\title{
Heterogramme in hurritischen Texten aus Boğazköy und Nordsyrien
}

\begin{abstract}
The article discusses the use of heterograms in Hurrian texts from Anatolia and Northern Syria of the second millennium BC. The frequency of Sumero- and Akkadograms is examined through a variety of Hurrian text genres. Most texts of religious content, which include rituals, festivals, or myths, exhibit only a small number of logograms and determinatives. Hurrian mantic texts from Hattuša and Emar offer an exception to these findings, in that they make use of a comparatively large amount of heterograms. In order to interpret these data one should consider a number of parameters, such as the scribes' level of foreign language acquisition, the texts' possible addressees, and the significance of text genres and styles. For comparison, a short overview of heterographic writing in Urartian texts has been provided.
\end{abstract}

Keywords: Hurrian cuneiform texts; Anatolia; Northern Syria; Urartian inscriptions; scribal traditions.

Von den Hethitern sind zahlreiche Tontafeln mit Keilschrifttexten überliefert, die sich in Archiven des 2. vorchristlichen Jahrtausends vorrangig in der hethitischen Hauptstadt Hattuša fanden. Auch an anderen Orten Anatoliens traten - in unterschiedlicher Anzahl - Tontafeln zu Tage, darunter in Maşat Höyük - Tapikka (Alp 1997), Kuşaklı - Šarišša (Wilhelm 1997), Ortaköy - Šapinuwa (vgl. De Martino - Süel 2015, v.a. 7f. mit Verweis auf weitere Literatur), Kayalıpınar - Šamuha (Rieken 2019), um an dieser Stelle nur die Wichtigsten zu nennen. Neben hethitischen Texten wurden auch solche in luwischer, palaischer, hattischer, akkadischer, sumerischer und hurritischer Sprache in den Archiven Hattušas gefunden. Im Folgenden stehen die hurritisch-sprachigen Texte und hethitischen Texte mit einzelnen hurritischen Abschnitten im Vordergrund, deren Überlieferung mit der sog. Mittelhethitischen oder Vorgroßreichszeit beginnt und sich bis zum Ende des hethitischen Großreichs verfolgen lässt (ca. 1500 bis 1180 v. Chr.). Sie wird u.a. mit einem starken hurritischen Einfluss auf das Hethiterreich in Verbindung gebracht, welcher aus dessen Auseinandersetzungen mit und um Kizzuwatna, der Region um das heutige Adana, resultierte. Diese Auseinandersetzungen kulminierten in der Integration Kizzuwatnas in das Hethiterreich im 15. Jh. v. Chr. Weiteren hurritischen Einfluss lieferte die Eroberung Mittanis unter Šuppiluliuma I. zu Beginn der Großreichszeit und vor allem die Hochzeit des Königs Hattušili III. mit Puduheba, der Tochter des Priesters der Šaušga von Lawazantija. Puduheba sorgte auch dafür, dass Abschriften des hišuwa-Festes aus Kizzuwatna in Hattuša angefertigt wurden (vgl. unten).

Die meisten der in Hattuša gefundenen hurritischen Texte sind dem religiösen Sektor zuzuschreiben. Dazu zählen einsprachig hurritische Fest- und Beschwörungsritualtexte, hurritische Rezitationen in hethitischen Ritual- und Festbeschreibungen, mythologische Texte, Gebete, Omina und hurritisch-hethitische Bilinguen, darunter das „Lied der Freilassung“, mythologische Erzählungen um die Götter Teššob und Allani ebenso wie die ansonsten im Alten Orient nicht belegte Gattung der Parabeln ${ }^{1}$. Weitere hurritische Texte, darunter lexikalische Listen, religiöse Texte, Omina und Briefe, stammen aus verschiedenen Orten Nordsyriens und

\footnotetext{
${ }^{1}$ Vgl. von Dassow 2013, bes. 131-146 zur Frage verschiedener Versionen und Fassungen des Textes / der Texte.
} 
Mesopotamiens. Zu nennen sind z.B. die aus dem 3. Jt. stammende Gründungsinschrift des Tiš-atal vermutlich aus Urkeš, Beschwörungen aus Larsa und Mari, ein Brieffragment aus Larsa, der große Mittani-Brief aus el-Amarna in Ägypten, hurro-akkadische Texte aus Alalah, Qațna und Nuzi, sowie Vokabulare und religiöse Texte aus Ugarit und lexikalische Listen und Omina aus Emar sowie ein Fragment aus Tigunanu ${ }^{2}$.

Im Folgenden sollen zunächst Abschnitte der verschiedenen hurritisch-sprachigen Textgattungen vorrangig aus Hattuša vorgestellt werden, in denen Heterogramme, d.h. Akkadound Sumerogramme, belegt sind. Unter Heterogramm wird dabei "a general cover term for Sumero- and Akkadogramms in Hittite texts" verstanden (Kudrinski - Yakubovich 2016: 64). Die Zusammenschau möchte unter Berücksichtigung weiterer hurritischer Texte, vor allem aus Nordsyrien, Überlegungen in Bezug auf die Verwendung von Heterogrammen in hurritisch-sprachigen Texten formulieren.

Zunächst soll ein Blick auf die Fest- und Beschwörungsrituale der hurritischen Tradition geworfen werden. Begonnen werden soll mit einem Abschnitt des hišuwa-Festes. Dieses Fest zählt zu den großen Festen der Hethiter und lässt sich besonders mit der Großkönigin Puduheba, Gemahlin Hattušilis III., in Verbindung bringen, die es, aus ihrer kizzuwatnäischen Heimat stammend, in Hattuša bekannt machte, wie aus den Tafelunterschriften der Texte deutlich wird:

KBo 15.52+ (CTH 628; jH)

(Rs. VI 39') MUNUS.LUGAL f $p u-d u-h e ́-p a-a \check{-}-k a ́ n ~ k u-w a-p i ́(40 ')$ mUR.MAH-LÚ-in GAL.DUB.SAR MEš

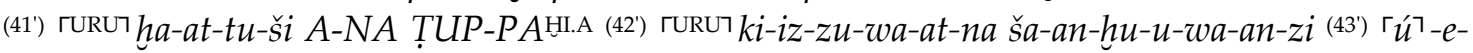

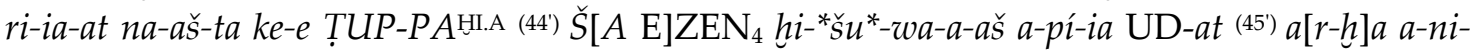
ia-at

„Als Königin Puduheba den Obertafelschreiber UR.MAH-zidi beauftragte, in Hattuša nach Tafeln aus Kizzuwatna zu suchen, hat er diese Tafeln des hišuwa-Festes an jenem Tage kopiert. “3

Das Fest, das wohl Waffenglück für den König und damit Leben und Wohlergehen für die königliche Familie und das ganze Land sichern sollte, ist in zahlreichen Abschriften überliefert, die von seiner Bedeutung für die Königsfamilie in Hattuša zeugen dürften. Bislang lassen sich knapp 500 Fragmente ca. 310 Tafeln zuordnen. Ca. 25 davon sind in mittelhethitischer Zeit niedergeschrieben worden, der Großteil in junghethitischer Zeit und einige wenige (ca. 15) sind als spätjunghethitische Niederschriften zu klassifizieren ${ }^{4}$.

Generell weisen die hurritischen Abschnitte des folgenden Ausschnitts (Vs. III 22-24, 2729, 33f., 35f. - hier nicht kursiv gesetzt) im Vergleich zu den benachbarten hethitischen Passagen keine Akkado- und Sumerogramme auf:

KBo 15.48+ (CTH 628; jH)

(Vs. III 18) $n u-z a$ LúSANGA GAL A da-a(Rasur)-i (19) an-da-ma-kán GIšEREN ki-it-ta-ri (20) nu wa-atar PA-NI ḩu-u-up-ru-uš-h̆i (21) la-ḩu-u-wa-i nu me-ma-a-i(22) a-zu-ma-aš-ši-ia šu-mi-pu-na (23) le-el-lu-re-en-na a-ba-te-in-na (24) ti-ia-ri-in-na ma-nu-uz-zu-un-ni ${ }^{2}$ (25) $n u-u s ̌-s ̌ a-a n ~ w a-a-$ tar GIŠEREN-za LUGAL-i (26) ŠUHI.A-*aš* la-huu-u-wa-i $n u$ me-ma-a-i (27) a-zu-ma-aš-ši-ia šu-u-

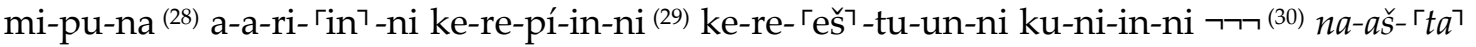

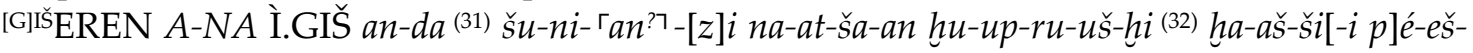

${ }^{2}$ Siehe für einen Überblick Wegner 2007: 24-30, und zu dem Text aus Tigunanu Salvini 1996: 123-128.

${ }^{3}$ Siehe u.a. Waal 2015: 441.

${ }^{4}$ Vgl. zur Datierung und Textzusammenstellung S. Košak, hethiter.net/: hetkonk (v. 1.993) sub CTH 628 (Zugriff 29.3.2020). 
ši-ia-zi ${ }^{(33)} n u$ me-ma- $\ulcorner a-i\urcorner$ a-har-re-eš ge-lu-uš ${ }^{(34)}$ te-e-a EGIR-ŠÚ $-m a 5$ NINDA.SIGMEš pár-šiia (35) $n u$ me-ma-a-i a-a-nu-iš har-ša-iš (36) ge-lu-uš te-e-a nu-kán A-NA NINDA.SIGMEš (37) $a$ na-a-hii da-a-i na-at-kán A-NA İ.GIŠ (38) an-da šu-un-ni-ez-zi

„Der SANGA-Priester nimmt sich einen Becher Wasser. Darin liegt Zedernholz. Er gießt Wasser vor dem Räucherständer aus und spricht: „... die Lelluri, Abadi, die Spindel(?) von Manuzzi. “5 (§-Strich) Das Wasser mit Zedernholz gießt er dem König auf die Hände und spricht: „... der böse(?) kirpinni-Mensch, der k.-ische kereštunni-Mensch.“ (§-Strich) Dann tunkt er das Zedernholz in Öl und wirft es auf den Räucherständer auf dem Herd und spricht: „Möge er (der König?) vollkommen heil sein durch den Weihrauch!“6 Anschließend bricht er fünf Fladenbrote und spricht: „Möge er (der König?) vollkommen heil sein durch dieses Brot! “7 Von den Fladenbroten nimmt er eine Kostprobe und tunkt sie in das Öl.“8

Diese hurritischen Abschnitte weisen keine heterographischen Schreibungen auf. Zieht man die weiteren, dem hišuwa-Fest zuzuordnenden Texte in Betracht, finden sich in den hurritischen Abschnitten nur die Sumerogramme DINGIR und $\mathrm{TU}_{7}$, die beide als Determinative Verwendung finden ${ }^{9}$.

Eine vergleichbare Situation findet sich in den den Ritualen der AZU-Priester zuzurechnenden Texten, die große Ähnlichkeiten mit den Texten des hišuwa-Festes in Bezug auf rituelle Handlungen und Rezitationen aufweisen und ebenfalls aus einer südostanatolischhurritischen Ritualtradition stammen. Beschrieben wird in ihnen häufig die Verehrung der beiden wichtigsten hurritischen Götter, des Wettergottes Teššob und seiner Gemahlin Hebat. Als Beispiel soll hier ein Abschnitt einer Textgruppe dienen, die als „Trankopfer für den Thron der Hebat“ (CTH 701) bezeichnet werden:

KBo 21.33+ (CTH 701; $\mathrm{mH})$ Vs. I

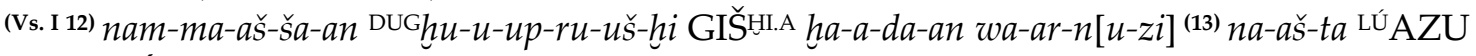
BE-EL SÍSKUR PA-NI DINGIR-LIM an-da pé-hu-te-[ez-zi] ${ }^{(14)} n u-k a ́ n ~{ }^{\mathrm{DUG}} a-a h-r u-u s ̌-h i-i a-a z$ GIšEREN ša-ra-a da-a-i ${ }^{(15)} n a-a t$ A-NA BE-EL SÍSKUR pa-a-i BE-EL SÍSKUR UŠ-KÉ-EN (16) LÚAZU-ma hur-li-li ku-un-zi-ip zu-uz-zu-ma-a-ki-ip KI.MIN $m[e-m a-i]$ רדר (17) $n u-z a$ LÚAZU GÙB-la-az ki-iš-ra-az MUŠEN.GAL da-a-i ku-un-na-za-ma-k[án] ${ }^{(18)} k i-i s ̌-r a-a z{ }^{*} \mathrm{DUG}^{*} \mathrm{DUG} a-a h-$ $r u-u s ̌-h i-i a-a z$ GIšEREN ša-ra-a da-a-i(19)$n a-a s ̌-t a$ İ.GIŠ $A-N A$ DUGGAL ME-E an-da za-ap-nu-zi $n u-z a \breve{S} A$ Dhé-p[át] ${ }^{(20)} k a-a t-k i-s ̌ a ~ i r-h a-a-i z-z i$ nu-uš-ša-an GIšEREN kat-ta A-NA DUGGA[L ME$E]^{(21)} d a-a-i n u-z a$ DUGGAL ME-E ša-ra-a da-a-i nu wa-a-tar A-NA DINGIR-LIM (22) me-na-ah-


a-i a-ah-ra-a-i ú-na-am-ma Giške-e-eš-hé-ep-we ${ }_{e}{ }^{(25)}$ ke-el-t[e]-i-e-ni a-am-ba-aš-ši-ni ke-e-lu

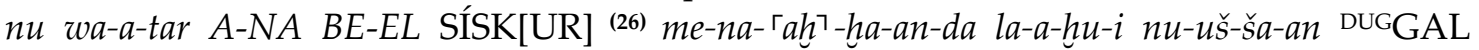
$M E-E$ kat-ta ${ }^{(27)} A-N A$ GIšBANŠUR AD.KID da-a-i $i$ ר

„Ferner verbrennt er auf dem Räucherständer getrocknete Hölzer. Der AZU-Priester bringt den Ritualherren vor die Gottheit, nimmt von der Räucherschale das Zedernholz

\footnotetext{
${ }^{5}$ Bei den Formen dieser Worte handelt es sich wohl um Absolutive - vgl. den später folgenden Spruch III 41-45, der von Schwemer 1995: 97, wie folgt übersetzt wird: „Diese Kostprobe soll zufriedenstellen die Lelluri, Abadi, die Spindel(?) von Manuzi; das Brandopfer für ihr Wohlbefinden aber möge sie sehr angenehm machen.“ Vgl. Hutter - Braunsar-Hutter 2016: 295 Anm. 5.

${ }^{6}$ Siehe zu dieser Konstruktion Campbell 2015: 141. Vgl. Hutter - Braunsar-Hutter 2016: 295.

${ }^{7}$ Siehe Campbell 2015: 141 und Hutter - Braunsar-Hutter 2016: 295.

${ }^{8}$ Nach Wegner - Salvini 1991: 33f. (ohne Übersetzung der hurritischen Passagen).

${ }^{9}$ Wegner - Salvini 1991: 36, lesen GIŠ als Determinativ. Der Abschnitt ist jedoch nicht GIšzé-er-ra-e, sondern ke-e-e]z-zé-er-ra-e zu lesen (KBo 27.198 8' - vgl. VSNF 12.22 8'!: ke-e-ez-zi-ri-ra-e (in ChS I/4 nicht berücksichtigt)).
} 
hoch und gibt es dem Ritualherrn. Der Ritualherr verbeugt sich. Der AZU-Priester spricht „Dein kunzi-, dein zuzzumaki- dito.“ auf hurritisch ${ }^{10}$. (§-Strich) Der AZU-Priester nimmt sich mit der linken Hand eine Gans, mit der rechten Hand aber nimmt er Zedernholz aus der Schale hoch und träufelt Öl in den Becher Wasser. Er beopfert das katkiša der Ištar. Er legt das Zedernholz in den Becher Wasser, nimmt den Becher Wasser hoch und gießt Wasser der Gottheit gegenüber aus. (§-Strich) Auf hurritisch spricht er folgendermaßen: „Wasch dir, Hebat, deine Hand mit Wasser und Weihrauch! Komm! Sei zufrieden gestellt durch keldi- und ambašši-Opfer deines Throns. “"11 Und er gießt das Wasser dem Ritualherrn gegenüber aus und stellt den Becher Wasser auf den Rohrflechttisch hinab. “12

Auch in diesen hurritischen Abschnitten werden bis auf KI.MIN ${ }^{13}$ und die Determinative DINGIR und GIŠ keine Heterogramme verwendet. Zieht man die weiteren, der CTH-Nr. 701 zuzuschreibenden Texte in Betracht ${ }^{14}$, zeichnet sich ein dem hišuwa-Fest vergleichbares Bild. Es finden sich die Sumerogramme DINGIR, GIŠ, DUG und URU als Determinative, die Heterogramme KI.MIN, URU, DINGIR ${ }^{\mathrm{MEŠ}}$ sowie GAŠAN und die logographischen Schreibungen von Götternamen IŠKUR, 10, 30, UTU und IŠTAR.

Die Beobachtung, dass hurritisch-sprachige Texte nur eine sehr begrenzte Anzahl von Sumero- und Akkadogrammen aufweisen, lässt sich auch für andere Textgattungen machen. So weist der hurritische Teil der hethitisch-hurritischen Bilinguen kaum Heterogramme auf; zu nennen sind die Determinative DINGIR, URU und der Personenkeil m sowie die Sumerogramme IŠKUR, IGI und $\mathrm{GU}_{4}{ }^{15}$. Zu bemerken bleibt, dass auch das Hethitische relativ wenig Heterogramme aufweist (so z.B. in KBo 32.14), ein Umstand, der der mittelhethitischen Niederschrift des Textes geschuldet sein dürfte ${ }^{16}$.

Ähnliches gilt für die hurritischen mythologischen Texte, die insgesamt nur sehr fragmentarisch überliefert sind. In diesen sind es vorrangig Namen, die heterographisch geschrieben werden: So finden sich die Akkadogramme DÉ-A und DIŠTAR sowie die Sumerogramme ${ }^{D} A . A$, DIŠKUR, DGIŠ.GIM.MAŠ, D10, DUTU, D30, LUGAL.GI. Des Weiteren sind die Determinative DINGIR, URU, KUR, m, f, GIŠ, GU, LÚ, UZU, MUŠ, die Sumerogramme DINGIR, EN, GU ${ }_{4}$, HUUR.SAG, İR, KASKAL, Ì.GIŠ, LÚ, SI.GAR, ŠU.GI, URU, SUKKAL, ŠÚ.A sowie die akkadographischen Komplementierungen $-I A$ und $-\breve{S} U$ in den mythologischen hurritischen Texten belegt $^{17}$.

Eine Ausnahme bilden die Fragmente, die den hurritischen mantischen Texte zuzurechnen sind. Überliefert sind Texte, die der Vorzeichenschau aus dem Verhalten eines Opfertieres während der Schlachtung oder dem Aussehen der Leber gewidmet sind. Zwei Texte beschäftigen sich darüber hinaus mit Missgeburts- und astrologischen Omina. In ihnen finden ver-

${ }^{10}$ Zur Analyse des Spruches vgl. Görke - Lorenz 2018/19: 50 sub Beispiel 6. Anders Neu 1996: 359: kunz=i=b entspricht akk. UŠKÊNN “er verbeugt sich”.

${ }^{11}$ Zur Analyse des Spruches vgl. Schwemer 1995: 90; zur Übersetzung „waschen“ von ašš- siehe Richter 2012: 51, mit weiterer Literatur.

${ }^{12}$ Vgl. Salvini - Wegner 1986: 38-41.

${ }^{13}$ Durch KI.MIN werden vorrangig ab der mittelhethitischen Zeit in verschiedenen Textgruppen v.a. Verben, Sätze und Abschnitte kurzgefasst wiederholt. Das Heterogramm KI.MIN ist dabei als technischer Marker, vergleichbar den deutschen Gänsefüßchen, zu verstehen und sollte daher am ehesten mit „dito“ übertragen werden. Siehe Görke - Lorenz 2018/19.

${ }^{14} 152$ Tafeln / 268 Fragmente nach S. Košak, hethiter.net/: hetkonk (v. 1.992) - 9.9.2019. Vgl. ChS I/2.

${ }^{15}$ Nach Neu 1996.

${ }^{16}$ Zur Datierung siehe Neu 1996: 3-7.

${ }^{17}$ Nach Salvini - Wegner 2004. 
gleichsweise viele Heterogramme Verwendung, eine Tatsache, die mit der Übernahme der betreffenden Techniken aus Mesopotamien in Zusammenhang stehen dürfte ${ }^{18}$. Mantische termini technici werden in den hurritischen Omen-Texten sumerographisch, wie aus mantischen Texten Babyloniens bekannt, geschrieben. Als Beispiel dafür kann die Bezeichnung eines Leberteils angeführt werden, der in akkadischen Texten akkadisch mazzāzu, ab dem Ende der altbabylonischen Zeit sumerographisch KI.GUB „Standort“, tituliert wird ${ }^{19}$. In dem hurritischen Text KBo 33.135 r. Kol. 1', 3', 9' aus Boğazköy (de Martino 1992: 31) findet sich die Schreibung KI.GUB-hé-ni mit der hurritischen Komplementierung -hé-ni des Sumerogramms KI.GUB für šintahe=ni „am Standort“. Bei der Übernahme der hurritischen Texte nach Zentralanatolien nutzten die Hethiter die sumerographische Schreibung in ihren (wenig belegten) Omentexten und verwendeten daneben auch die entsprechenden hurritischen Termini. Das ist z.B. erkennbar in KBo 10.7, einem Eingeweideomen, das in Rs. IV 4 die heterographische Schreibung A-NA KI.GUB iš-tar-na ,auf dem Mittelpunkt des Standortes“ aufweist, in Rs. IV 9 und 12 hingegen den hurritischen Terminus šintahi: 9f.: [ták-ku-uš-ša-a]n ši-in-ta-a-hri an-da [ ] $k i$-ša-ri „[Wenn] auf dem Standort [ ] entsteht“ (Riemschneider 2004: 28, 32). Die vorherrschende Rolle, die die mesopotamische Eingeweideschau besonders in Bezug auf Leberschauomina spielte, wird in der Anzahl vor allem der Sumerogramme in hurritischen Omentexten aus Hattuša deutlich. So weisen die insgesamt nur 45 hurritischen mantischen Textfragmente die Determinative LÚ, GIŠ, D, m und URU, die Akkadogramme IZBU, RUQQU, TIRANU und IŠTAR sowie die Sumerogramme ARAD, BA.ÚŠ, DAGAL, DIŠ, DINGIR, DÙG.GAN, DUMU.É.GAL, É, EN, É.EN.NU.UN, EGIR, ÉRIN, GEŠTU, GIG, GÍR, GÚ, GIŠGU.ZA, GÙB, IGI, IM, ITU, KA, KAxU, KÁ.É.GAL, KASKAL, KI.A, KI.GUB, KUR, LÚKÚR, LÀL, LÍL, LÚ, LU-

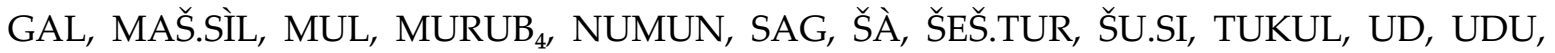
URU, ZAG, ZÍ, UTU, EN.ZU auf ${ }^{20}$.

Abschließend zu den hurritischen Texten aus Anatolien soll ein Text aus Ortaköy vorgestellt werden, der eine Parallele zu Tafeln des itkalzi-Rituals aus Boğazköy enthält. Die mittelhethitische Tafel ist bis auf Determinative wie f für Frau und das Sumerogramm DINGIRMEŠ "Götter" syllabisch geschrieben und reiht sich damit nahtlos in die hier bereits vorgestellten Beispiele ein. Inhaltlich enthält die Tafel bzw. ihr erhaltener Teil der Vs. I und Rs. IV hurritische Rezitationen, die im Zuge der Reinigung der im Text genannten Königin Taduheba evtl. anlässlich ihrer Hochzeit mit Tuthaliya II. vorgetragen wurden. Beispielhaft soll das folgender Abschnitt illustrieren:

Or. 90/1494 + Or. 90/1665 + Or. 90/1707 Rs. IV (mH; CTH 777)

(Rs. IV 17') aš-hu-ši-ik-ku-un-ni-bi fta-du-h̆[é-ba-a-bi i-ti] (18') pa-a-hii pa-a-ši ir-te kar-ši ha-ap-zu[-u-ri ] (19') i-ti it-ni šum-mi tar-šu-wa-an-ni-bi [ ] (20') aš-ḩu-ši-ik-ku-un-ni-ni-bi fta-du-hé-pa-a-b[i] (21') še-ḩa-lu-la-a-eš it-ku-la-a-eš ga-aš-lu-la-a[-eš ] (22') e-ku-uš-šu-laa-eš aš-huu-ši-ik-ku-un-ni (23') a-a-bi DINGIRMEŠ-a-a-ša

„Der Ritualherrin Taduh[eba Körper,] Kopf, Mund, Zunge, Lippe, Spuc[ke,] Körper, der ganze Körper der Person de[r] Ritualherrin Taduheba soll klar, rein, stark, sauber werden, die Ritualherrin vor den Göttern!“(de Martino - Süel 2015: 58f., 64f.)

Eine Ausnahme in Hinblick auf die Verwendung von Heterogrammen bildet der Kolophon dieses Textes. Er ist auf Hethitisch verfasst und gleicht den Kolophonen hethitischer Texte (vgl. dazu weiter unten):

\footnotetext{
${ }^{18}$ So bereits de Martino 1992: 4.

${ }^{19}$ Vgl. Meyer 1987: 56f. Für Beispiele siehe CAD M 235b sub 2.

${ }^{20}$ Vgl. dazu das Glossar in de Martino 1992: 129-138.
} 
Or. 90/1494 + Or. 90/1665 + Or. 90/1707 Rs. IV 28'-31' (mH; CTH 777):

DUB 3.KAM Ú-UL QA-T[I ŠA SÍSKUR it-kal-zi-ia-aš]

$a$-iš [š] u-up-pí-ia-[ah-hu-wa-aš ANA DUTUšs-at-kán]

KAxU-az pa-ra-a [I-NA URU zi-it-ha-ra]

I-N $[A]$ BURU $_{14} a-[n i-i a-u-e n]$

„3. Tafel, (Ritual) nicht beend[et; des Rituals des itkalzi-] und des Mund-Rein[igens;] auf

Anweisung [der Majestät] hab[en wir es in Zithara] im Sommer aus[geführt.]“21

Nicht ausführlich kann an dieser Stelle auf das Phänomen der Vollkomplementierung eingegangen werden, das sich vereinzelt in hurritischen Texten beobachten lässt. Darunter wird verstanden, dass syllabisch geschriebenen Wörtern das Determinativ gleicher Bedeutung beigefügt wird. Als Beispiel dafür können im Hurritischen Schreibungen von „Gott“ und „Götter“ im Mittani-Brief angeführt werden: z.B. Mit I 78: DINGIR.MEše-e-en-na-šu-uš. Dabei sind die Pluralformen alle mit dem Determinativ DINGIR.MEš versehen (I 78, II 52, II 76,77, IV 64, 65, IV 117, 118), während zwei der drei Singularformen kein Determinativ aufweisen (I 87, I 195 vs. III 98 ${ }^{22}$. Das Phänomen ist allerdings nicht auf das Hurritische beschränkt. Bereits im althethitischen Text KBo 17.3+ (CTH 416) Rs. IV 12 findet sich die hethitische Schreibung GIšta- $a$ ru „Holz“ parallel zu dem in KBo 17.1+ Rs. IV 16 belegten GIŠ-ru (cf. C. Montuori (ed.), hethiter.net/: CTH 417 (TX 8.2.2017, TRit 24.7.2015) § 43 Kolon 237 - Zugriff 19.11.2019). Formen von GIš.HURgulzattar „Tafel“ finden sich in KUB 50.6+ (CTH 569) Rs. III 12, 18 (jH), sowie in KBo 11.1 (CTH 382) Vs. 41 (jH). In Bezug auf eine Schreibung Ďši-ú-na-aš oder Ďši-ú-ni vgl. CHD Š 463 mit der Möglichkeit einer Lesung DINGIR-LIM-ú-na-aš oder DINGIR-LIM-ú-ni (siehe dagegen Starke 1979: 55f.). Als keilschriftluwischer Beleg ließe sich KÁaštummant- „Tor“ in KBo 29.31 (CTH 763) Rs. IV 5' (jH) anführen ${ }^{23}$.

Insgesamt zeigen damit sowohl einsprachig hurritische Texte als auch hurritischsprachige Abschnitte in den hethitischen Texten aus Boğazköy und Ortaköy weit weniger Heterogramme, als man von dem Gros der hethitischen Texte gewöhnt ist. Dies gilt zumindest für die Textgruppen der Fest- und Beschwörungsrituale, der hurritisch-hethitischen Bilinguen und der hurritischen mythologischen Texte. Einzig die hurritischen mantischen Texte weisen einen höheren Anteil an heterographischen Schreibungen auf.

Differenziert man die einzelnen Gruppen nach der Art der hurritischen Abschnitte, ist in Bezug auf die Fest- und Beschwörungsrituale zu konstatieren, dass es sich hierbei in allen Fällen um Rezitationen handelt, die zu den auf hethitisch notierten Ritualhandlungen gesprochen werden. Die mythologischen Texte sowie die Bilinguen geben dagegen auch Erzählungen auf Hurritisch wieder ${ }^{24}$.

In allen Fällen überwiegt die Verwendung von Sumerogrammen gegenüber Akkadogrammen $^{25}$ sowie der Gebrauch von Sumerogrammen als Determinative. Häufige Determinative sind dabei D, URU, GIŠ, m, f, häufige Sumerogramme u.a. sumerographisch geschriebene Götternamen wie IŠKUR oder UTU, aber auch die Notation einer namentlich nicht genannten

${ }^{21}$ De Martino - Süel 2015: 59, 65 mit Ergänzungen u.a. aus FHG 21+ Rs. IV 38'-41' (ChS I/1 Nr. 6, S. 77), KBo 23.6+ Rs. IV 36'-38' (ChS I/1 Nr. 5, S. 65).

${ }^{22}$ Belege nach Dietrich, Mayer 2010: 240. Die dort getroffene Aussage „Ohne Determinativ ist êni= Apposition zu einem Götternamen. “ ist zu relativieren, da auch der Beleg in III 98 und vermutlich die in I 78, II 77, IV 118 als Appositionen mit Determinativ auf gefasst werden dürften. Zu einer Form Den-na- in einem Text aus Emar siehe Fischer 2017: 36.

${ }^{23}$ Ich danke J. Lorenz für diese Hinweise.

${ }^{24}$ Vgl. dazu auch ein hurritisches Fragment aus Kayalıpınar (Wilhelm 2019: 197-200).

${ }^{25}$ Auch in hethitischen Texten überwiegt die Verwendung von Sumerogrammen gegenüber Akkadogrammen. 
Gottheit mit DINGIR oder die vereinfachte Schreibung von geographischen Begriffen wie ÍD „Fluss“, HUR.SAG „Berg“ und URU „Stadt“ gegenüber den hurritischen Äquivalenten. Akkadogramme in mythologischen Texten umfassen im Wesentlichen Götternamen wie ǏSTAR oder $E A$ und vor allem Fachbegriffe der Eingeweideschau in den mantischen Texten.

Eine zeitliche Differenzierung zwischen der Verwendung von Heterogrammen in mittel-, jung- und spätjunghethitischen Niederschriften ist nicht erkennbar. Die bislang angeführten Beispiele stellen Niederschriften der Großreichszeit dar (hišuwa-Fest CTH 628, mythologische Texte - siehe dazu Salvini - Wegner 2004: 13f.), die ebenso wenige Heterogramme aufweisen wie die Rituale der AZU-Priester (CTH 701), die hurritisch-hethitische Bilinguen oder der zitierte Texte aus Ortaköy, die beide in der mittelhethitischen Schriftperiode niedergeschrieben wurden.

In einem Vergleich mit anderen fremdsprachigen Texten in den Archiven Hattušas, also hattischen, palaischen und luwischen Texten, ist festzustellen, dass auch diese eine Präferenz für syllabische Schreibungen erkennen lassen. Wie bei den hurritischen Passagen handelt es sich vorwiegend um Rezitationen oder mythologische Erzählungen ${ }^{26}$. In Bezug auf die palaischen Textpassagen geht Kudrinski davon aus, dass die Verwendung der Heterogramme nach dem Willen des Schreibers bei Diktat des Textes erfolgte, ohne dass der Schreiber besondere Palaisch-Kenntnisse gehabt hätte (Kudrinski 2017: 245f.). Im Gegensatz dazu dürfte davon auszugehen sein, so Kudrinski, dass die Schreiber des Luwischen im Wesentlichen mächtig waren, was zu einem vermehrten Gebrauch von Heterogrammen, insbesondere Sumerogrammen, im Vergleich mit der Niederschrift der palaischen Passagen, geführt habe (Kudrinski 2017: 246-248).

Wie oben bereits angemerkt, weisen auch einsprachig hurritische Texte keine hurritischen Kolophone auf. Allgemein dürften alle Kolophone hethitisch verstanden worden sein ${ }^{27}$, auch wenn manche vollständig heterographisch geschrieben sind und die dahinterstehende Sprache damit nicht bestimmt werden kann. Als Beispiel dafür mag der Text KUB 47.2 (Hurritische Version des Kešše-Epos) dienen: lk. Rd. 1-3 DUB 14.KAM SÌR mke-eš-še NU.TIL „14. Tafel (des) Liedes (von) Kešše. Nicht beendet. “ Hinweise auf fremdsprachige Kolophone lassen sich nur in Ausnahmefällen finden. So dürfte es sich dabei teilweise um die Titel oder Anfangswörter fremdsprachiger Rezitationen handeln, z.B. KBo 25.135 rev. 7' [ tup-p]í? 2.KAM ša-i-šumi-da-a-a[n] ${ }^{28}$ (hattisch - cf. Waal 2015: 516 mit Lesung $\breve{S} A$ i-šu-mi-na-a-a[n ) oder KUB 28.83 rev. 3' ] 1 SİR i-waa-ah-ru-wa a-aš-da-ak-ši-du ${ }^{29}$ (hattisch - Waal 2015: 516). Ob das auch auf den Kolophon des hurritischen Fragments KBo 8.143 rev 15' ] [DINANNA7 šu-pal-wu ${ }^{\prime}$ da-a-iaa-na-a-x[ (Waal 2015: 533 - cf. Trémouille 2005: 23 mit einer Lesung ] 「DINANNA7 ŠU BAL$\mathrm{wu}_{\mathrm{u}}$ da-a-ia-a-na-a-x[ ) zutrifft, muss derzeit offen bleiben.

Vergleicht man diesen Befund einer Präferenz syllabischer Schreibungen in hurritischen Texten aus Anatolien mit entsprechenden Analysen hurritischer Texte anderer Fundorte, ist festzustellen, dass auch diese überwiegend syllabisch geschrieben sind: Die vorrangig religiösen Texte aus Ugarit $^{30}$ in syllabischer Schrift weisen kaum Heterogramme auf, sind aber zum

${ }^{26}$ Hethitische Rezitationen wie z.B. die Anrufungen des Ambazzi-Rituals CTH 391.1 und .2 oder die der Baurituale CTH 413.1 und 414.1 sind ähnlich mit Heterogrammen gespickt wie hethitische Ritualbeschreibungen.

${ }^{27}$ Waal 2015: 506: "In general, the colophons of texts in other languages follow the same pattern as comparable texts composed in Hittite: the colophons of the festival and ritual texts are generally the same as the festival and ritual texts listed CTH 591-725 and CTH 390-500 respectively. The same applies to the prayers, omen texts and invocations."

${ }^{28}$ Soysal 2004: 510: Eventuell als šai=šumin=an für *tai=*tumin=an(?) zu verstehen.

${ }^{29}$ Soysal 2004: 389: Verbales $a=s ̌=d a=k=s ̌ i t(=) u\left[\right.$ für $a=\check{s}=* t a={ }^{*} k a=s ̌ i t(=) u(?)$.

${ }^{30}$ Siehe dazu Laroche 1968: 447-544, v.a. 462-496. Vgl. auch Dietrich-Mayer 1999: 58-62. 
Teil nur sehr fragmentarisch überliefert. Auch die hurritischen Beschwörungen aus Mari sind syllabisch geschrieben ${ }^{31}$. Der Mittani-Brief als hurritisches Dokument par excellence enthält nur wenige Heterogramme, darunter die Determinative $D, m, f, K U R$, URU, MEŠ, die Sumerogramme DINGIRMEš, KUR, URU, GUŠKIN, $\mathrm{NA}_{4}$ ZA.GİN sowie das Akkadogramm șalmu ${ }^{32}$. Unter den in Emar gefundenen hurritischen Texten sind zahlreiche Leberschauomina, die ebenso wie die mantischen Texte aus Hattuša - eine Vielzahl v.a. an Sumerogrammen aufweisen. Beispielhaft sei auf den Text MSK 74.192a $+{ }^{33}$ verwiesen, einem mit 127 Zeilen sehr langen Text mit Leberschauomina, der alleine in den ersten 12 Zeilen 94 Sumerogramme verwendet (Salvini 2015 I: 98-104 mit einer Transliteration des Textes) ${ }^{34}$. Zu bemerken bleibt, dass besonders die wenigen Textfunde aus dem Jahr 1973 weit weniger heterographische Schreibungen aufweisen als das Gros der Texte, die im Jahr 1974 gefunden wurden ${ }^{35}$.

Berücksichtigt man für eine Auswertung auch die Einteilung von Texten gemäß der Gruppierung nach van den Hout 2002 in eine Gruppe A „Texte mit Duplikaten“ sowie eine Gruppe B „unica“ ${ }^{36}$, sind die hurritischen Rituale, mythologischen Texte sowie Parabeln mit vergleichsweise wenigen Heterogrammen der ersten Gruppe zuzuschreiben, während die mantischen Texte mit ihren zahlreichen Heterogrammen den unica zuzurechnen sind.

Als Erklärung für eine Auswertung dieser Beobachtungen wurde wiederholt das Fremdsprachenniveau der Schreiber angeführt. Salvini (1980: 166f.) vermutete, dass die hethitischen Schreiber des (h)išuwa-Festes die hurritischen Rezitationen Wort für Wort kopierten, ohne sie recht $\mathrm{zu}$ verstehen ${ }^{37}$. Auch Kudrinski bringt ein höheres Fremdsprachenverständnis als Argument für einen vermehrten Gebrauch von Heterogrammen vor (Kudrinksi 2017: 246, 249). Angenommen wird, dass Rezitationen syllabisch geschrieben wurden, um eine falsche Interpretation der Logogramme, und damit eine fehlerhafte Aussprache der dann wirkungslosen Rezitation zu verhindern ${ }^{38}$. Demgegenüber steht die Beobachtung, dass hurritische Texte auch außerhalb Anatoliens vorwiegend syllabisch geschrieben worden sind. Weiterhin dürften Schreiber in Anatolien als Experten auf ihrem Gebiet angesehen worden sein und besonders für hethitische Schreiber, die hurritische Werke ins Hethitische übersetzten, darf ein gewisses Verständnis der hurritischen Sprache vorausgesetzt werden (Hoffner 1992: 105; Klinger 2001; Corti 2007: 120f.). Insbesondere zu einer Zeit wie der mittelhethitischen, in der man von der

${ }^{31}$ Thureau-Dangin 1939, 1-28; Salvini 1988: 81.

${ }^{32} \mathrm{Vgl}$. die Umschrift in Dietrich-Mayer 2010: 27-53.

${ }^{33}$ Siehe Fischer 2017: 31 Anm. 6, zu einem Join mit MSK 74.238A.

${ }^{34}$ Siehe zu den in den hurritischen Emar-Texten gebrauchten Sumerogrammen den Thesaurus (Salvini 2015 II: 211-281).

${ }^{35}$ Siehe z.B. die Texte MSK 73.1034 oder MSK 73.1049+ (Salvini 2015 I: 57-59). Zur Einteilung der hurritischen Texte aus Emar in zwei Gruppen, von denen die erste im Wesentlichen die Textfunde aus dem Jahr 1973 und einige wenige aus dem Jahr 1974 umfasst, während die zweite die restlichen Textfunde von 1974 einschließt, siehe Fischer 2017, vor allem 32-40. Vgl. dort S. 36 auch die Bemerkungen zum Gebrauch von Logogrammen.

${ }^{36}$ Siehe van den Hout 2002: 864; zur Gruppe A sind demnach u.a. historische Texte, Verträge, Edikte, Instruktionen, Gesetze, Hymnen und Gebete, Festtexte, Ritualtexte, mythologische Texte, lexikalische Texte sowie fremdsprachige Texte zu zählen, während sich in der Gruppe B Briefe, Landschenkungsurkunden, hippologische Texte, Orakeltexte, Gelübde und einige administrative Texte finden. Für eine weitere Aufteilung in vier Gruppen siehe Waal 2015, bes. 173-175.

${ }^{37}$ Vgl. dazu Gordin 2015: 153 mit Anm. 631, der sich jedoch nicht in Bezug auf die Hurritisch-Kenntnisse der Schreiber Talmi-Teššub und Hulunabi festlegen möchte.

${ }^{38}$ Das hieße auch, dass die hurritische Lesung der Logogramme eindeutig gewesen sein muss. Kudrinski Yakubovich 2016 machen wahrscheinlich, dass Heterogramme im Hethitischen zusätzliche Informationen semantischer Art liefern könnten. Vgl. Kudrinski 2017. An dieser Stelle kann diesen Fragestellungen nicht weiter nachgegangen werden. 
Anwesenheit hurritischer Muttersprachler in Hattuša ausgehen kann, überzeugt mich das Argument des unkundigen Schreibers nicht. Erinnert werden soll in diesem Zusammenhang auch an den Schreiber mit dem hurritischen Namen Hubidi, von dem hurritische und hethitische Tafeln überliefert sind: ein hurritisches Gebet der Taduhepa an Teššub für das Wohl von Tašmi-Šarri ${ }^{39}$ und ein hethitisches Fragment eines militärischen Reinigungsrituals ${ }^{40}$. Die Frage nach dem Fremdsprachenniveau des Schreibers ist demnach kritisch oder zumindest differenziert zu betrachten.

Neben dem Fremdsprachenniveau des Schreibers gilt es auch, dasjenige des bzw. der Adressaten $\mathrm{zu}$ berücksichtigen. In diesem Zusammenhang sei an die Argumentation von B. Christiansen erinnert, die die Festritualtexte als Anweisungstexte verstehen möchte. Vergleichbar mit dem Missale Romanum der römisch-katholischen Messe von 1970 könnten sie den Priestern und anderen Kultakteuren dazu gedient haben, sich den Verlauf des Festes und die zugehörigen Riten einzuprägen, um sie korrekt auszuführen (Christiansen 2016: 41) ${ }^{41}$. Eine englischsprachige Anleitung für katholische Messdiener verweist darüber hinaus auf die korrekte Aussprache lateinischer Texte, ohne aber eine Übersetzung zu liefern. Nach Christiansen (2016: 53) wird dadurch wohl dem korrekten Vollzug der Riten ein höherer Wert beigemessen als deren Verständnis durch die Messdiener. Für die anatolischen Texte mag ein Blick auf die hurritischen mantischen Texte und Omina deutlich machen, dass diese, die durch ihren vergleichsweise häufigen Gebrauch von Heterogrammen (v.a. Sumerogrammen) herausstechen, gleich ob sie aus Hattuša oder Emar stammen, zur Gruppe der Unikatstexte gehören. Sie wurden also eher für einen konkreten Moment gebraucht und richteten sich sicher nur an Experten. Die Adressaten der anderen hurritischen Texte sind schwieriger zu bestimmen und sollen an dieser Stelle offen bleiben. Sicherlich dürfte eine syllabische Schreibung das Vorlesen von Texten auch bei Unkenntnis der jeweiligen Sprache erleichtert haben, was z.B. auf die palaischen Texte zutreffen könnte ${ }^{42}$.

Betrachtet man vergleichend die urartäischen Inschriften des 1. Jt. v. Chr., ist zunächst deutlich zu machen, dass diese einem anderen Textgenre, nämlich dem der Königsinschriften zuzurechnen sind. Heterogramme werden nach einer Durchsicht der urartäischen Texte in allen Inschriften, aber nicht in übermäßiger Zahl genutzt. Gehäuft treten sie in Elementen der Herrschaftstitulaturen sowie in Auflistungen von Beute oder Opfergaben auf. Vergleicht man die Verwendung von Akkadogrammen in parallelen akkadischen Versionen, liegt der Anteil in urartäischen Texten bei etwa der Hälfte. Als Beispiel dafür mag die Kelišin-Stele vom Ende des 9. Jh. v. Chr. dienen. In den teilweise ergänzten 41 Zeilen der urartäischen Version werden 46 Determinative (DINGIR, m, URU, KUR, MEŠ), 27 Sumerogramme (u.a. MAN, URUDU, $\mathrm{GU}_{4}$, KÁ, IM, UTU, DINGIR) sowie 8 Akkadogramme (DANNU, LIM, ME) genutzt. In dem ebenfalls teilweise ergänzten, 42 Zeilen umfassenden assyrischen Text werden 54 Determinative (DINGIR, URU, m, MEŠ) und 67 Sumerogramme (u.a. MAN, A, GAL, DUMU) verwendet (siehe zu den beiden Versionen der Kelišin-Stele Salvini 2008 I: 141-144; zu weiteren, allerdings nicht so gut erhaltenen urartäischen Inschriften mit assyrischen Parallelen siehe Salvini 2008 I: 497-508, Inschriften A 10-3, A 10-4, A 10-5). Metallobjekte mit urartäischer Beschrif-

${ }^{39}$ KUB 32.19+, CTH 778.8, mH; siehe ChS I/1 Nr. 41: 215-232; Singer 2002: 43f.; Gordin 2015: 109, 351.

${ }^{40}$ KBo 22.129+, CTH 428, mH; siehe Groddek 2008: 120f.; Gordin 2015: 109, 351.

${ }^{41}$ Auch Burgin 2019 konnte in seiner Untersuchung zu den althethitischen Tafeln des KI.LAM-Festes deutlich machen, dass verschiedene Angaben und Formulierungen darauf schließen lassen, dass die Texte für unterschiedliche Adressatengruppen verfasst wurden; allerdings beschäftigte er sich nicht mit Tafeln, die fremdsprachige Partien enthalten.

${ }^{42}$ Ich verdanke diesen Hinweis I. Yakubovich. Vgl. auch oben und Kudrinski 2017: 245-248. 
tung weisen recht wenige Heterogramme auf, was damit in Verbindung stehen könnte, dass die Namen als Besitzangaben meist syllabisch geschrieben werden (vgl. die Darbietung der Texte in Salvini 2012: 19-74). Etwas mehr Heterogramme zeigen einige urartäische Tontafeln, besonders, wenn es sich um Listen, in denen die Verteilung von Gütern an verschiedene Personen notiert wird (vgl. Salvini 2012: 122f.), oder andere Verwaltungstexte handelt (vgl. Salvini 2012: 122-150). Diese weisen teils bis auf die Namen nur Heterogramme auf (siehe dazu z.B. die Tafel CT An-1 aus Anzaf; siehe dazu Salvini 2012: 122f.; vgl. auch die Tafeln aus Toprakkale; siehe Salvini 2012: 145-150). Für das Urartäische kann damit festgehalten werden, dass die Verwendung von Heterogrammen textsortenspezifisch variiert. Darüber hinaus kann davon ausgegangen werden, dass Listen und Verwaltungstexte von Verwaltungsbeamten für Verwaltungsbeamte notiert wurden, man hier also in gewisser Weise von Experten mit identischem Kenntnisstand ausgehen kann. Urartäische Namen, und zwar Götter-, Orts- und Personennamen, werden fast ausschließlich syllabisch geschrieben (vgl. Salvini 2008 II: 258-372, oder auch Salvini 2012: 93-108, 159-163, 203-205) und der Gebrauch von Heterogrammen in Königsinschriften kann unter Beeinflussung der assyrischen Schreibtradition gestanden haben. Als Adressaten derselben dürften die einheimischen Urartäer gelten, für die ein problemloses Verständnis der Inschriften angenommen werden darf.

In einer Zusammenschau scheinen die hurritischen Texte aus Boğazköy in einer anderen Schreibtradition zu stehen als die hethitischen Texte. Da alle bislang auf uns gekommenen hurritischen Texte - mit Ausnahme der mantischen Texte - einen eher zurückhaltenden Gebrauch von Heterogrammen bezeugen, was sich im Übrigen bereits für die Tiš-AtalInschrift des beginnenden 2. Jt.s v. Chr. konstatieren lässt, fügt sich das Textmaterial aus Zentralanatolien (Hattuša und Ortaköy) sehr gut in den Befund ein. Besonders der Vergleich mit hurritischen Texten anderer Fundorte wie Ugarit oder Emar scheint darauf zu deuten, dass der Gebrauch von Heterogrammen in der Verschriftlichung der hurritischen (und anderssprachigen) Texte in Hattuša einer anderen Tradition zuzuschreiben ist und eher keine Rückschlüsse auf die Sprachkompetenz der Schreiber zulässt. Die Schreibungen in hethitischen Texten unter häufiger Verwendung von Heterogrammen, darunter auch Mischungen akkadischer und sumerischer Logogramme, teils mit hethitischen Komplementen, sind singulär im Alten Orient, ohne dass bislang eine grundlegende Erklärung für dieses Phänomen gefunden werden konnte $^{43}$. Ob bei den Hethitern ein Bewusstsein existierte, dass eine syllabische Schreibung die Tradierung und das Verständnis fremdsprachiger Texte auch bei zurückgehender allgemeiner Sprachkompetenz sicherstellen würde (und damit z.B. die korrekte Durchführung und Rezitation in Festen oder Ritualen) oder ob, wie von Kudrinski - Yakubovich 2016 angenommen, der Gebrauch von Heterogrammen bei der Verschriftlichung des Hethitischen semantisch bedingte Gründe hat, können nur weitere Studien erweisen.

\section{Abbreviations}

ChS I/1 - Haas 1984; ChS I/2 - Salvini-Wegner 1986; ChS I/4 - Wegner-Salvini 1991; ChS I/6 - Salvini-Wegner 2004.

${ }^{43}$ In manchen Texten finden sich Hinweise, dass sie laut vorgelesen wurden (vgl. HW2 $\mathrm{H} 101$ s.v. tuppi halzai„(Ton-)Tafel vorlesen, laut lesen“ mit Hinweis auf 11 Belege dafür, davon 6 Briefe, 3 Vertragstafeln und 2 Ritualtexte). Wird eine konkrete Person genannt, die vorlesen soll, ist es ein Schreiber. In Bezug auf die Frage nach der Sprachrealität des Hethitischen mit seinen zahlreichen Heterogrammen stellt sich daher die Frage, ob diese vom Schreiber wirklich hethitisch (vor)gelesen wurden (so z.B. Rieken 2011: 16) oder einfach nur gelesen wurden; vgl. Rieken - Yakubovich im Druck. 


\section{References}

Alp, Sedat. 1991. Hethitische Keilschrifttafeln aus Maşat-Höyük. Ankara: Türk Tarih Kurumu Basımevi.

Alp, Sedat. 1991b. Hethitische Briefe aus Maşat-Höyük. Ankara: Türk Tarih Kurumu Basımevi.

Burgin, James M. 2019. Functional Differentiation in Hittite Festival Texts. An Analysis of the Old Hittite Manuscripts of the KI.LAM Great Assembly, StBoT 65. Wiesbaden: Harrassowitz.

Campbell, Dennis. 2015. Mood and Modality in Hurrian. Winona Lake, Ind.: Eisenbrauns.

Christiansen, Birgit. 2016. Liturgische Agenda, Unterweisungsmaterial und rituelles Traditionsgut. Die hethitischen Festritualtexte in kulturvergleichender Perspektive. Studien zu den Boğazköy-Texten 60: 31-65.

Corti, Carlo. 2007. The so-called "Theogony" or "Kingship in Heaven". The name of the Song. Studi Micenei ed Egeo-Anatolici 49: 109-121.

De Martino, Stefano. 1992. Die mantischen Texte. Corpus der hurritischen Sprachdenkmäler: Texte aus Boğazköy, Vol. 7. Roma: Bonsignore Editore.

De Martino, Stefano, Aygül Süel. 2015. The Third Tablet of the itkalzi Ritual. Eothen Vol. 21. Firenze: LoGisma.

Dietrich, Manfried, Walter Mayer. 1999. The Hurrian and Hittite Texts. In: W. G. E. Watson, N. Wyatt (eds.). Handbook of Ugaritic Studies, HdO I/39: 58-75. Leiden / Boston / Köln: Brill.

Dietrich, Manfried, Walter Mayer. 2010. Der hurritische Brief des Dušratta von Mīttānni an Amenhotep III. Text Grammatik - Kopie, AOAT 382. Münster: Ugarit-Verlag.

Fischer, Sebastian. 2017. Erste Ergebnisse einer Untersuchung der hurritischen Emar-Texte. Mesopotamia 52: 31-53.

Gordin, Shai. 2015. Hittite Scribal Circles. Scholarly Tradition and Writing Habits, StBoT 59. Wiesbaden: Harrassowitz.

Görke, Susanne, Jürgen Lorenz. KI.MIN „dito“ in hethitischen Texten. Münchener Studien zur Sprachwissenschaft 72/1, 2018/19: 43-68.

Groddek, Detlev. 2008. Hethitische Texte in Transkription: KBo 22. Wiesbaden: Harrassowitz.

Haas, Volkert. 1984. Die Serien itkahi und itkalzi des AZU-Priesters, Rituale für Tašmišarri und Tatuhepa sowie weitere Texte mit Bezug auf Tašmišarri (Corpus der hurritischen Sprachdenkmäler I, Band 1). Roma: Multigrafica editrice.

Hoffner, Harry A. 1992. Syrian Cultural Influence in Hatti. In: Mark W. Chavalas, John L. Hayes (eds.). New Horizons in the Study of Ancient Syria: 89-106. Malibu: Undena Publications.

Hutter, Manfred, Sylvia Braunsar-Hutter. 2016. Kubaba im hišuwa-Fest. In: Sedat Erkut, Özlem Sir Gavat (eds.). ANTAHŠUM ${ }^{S A R}$ „Çĭ̆dem“ Eski Anadolu Araştımalarına ve Hititlere Adenmiş Bir Hayat (Studies in Honour of Ahmet Ünal): 293-302. Istanbul: Arkeoloji ve sanat yayınlar1.

Klinger, Jörg. 2001. Die hurritische Tradition in Hattuša und das Corpus hurritischer Texte. In: Thomas Richter, Doris Prechel, Jörg Klinger (eds.). Kulturgeschichten. Altorientalische Studien für V. Haas zum 65. Geburtstag: 197-208. Saarbrücken: SDV.

Kudrinski, Maksim. 2017. Heterograms in Hittite, Palaic, and Luwian context. Journal of Language Relationship 15: 239-250.

Kudrinski, Maksim, Ilya Yakubovich. 2016. Sumerograms and Akkadograms in Hittite: Ideograms, Logograms, Allograms, or Heterograms? Altorientalische Forschungen 43: 53-66.

Laroche, Emmanuel. 1968. Documents en langue hourrite provenant de Ras Shamra. In: Cl. Schaeffer (ed.). Ugaritica $V$. Nouveaux textes accadiens, hourrites et ugaritiques des archives et bibliothèques privées d'Ugarit: 448-544. Paris: Geuthner.

Meyer, Jan-Waalke. 1987. Untersuchungen zu den Tonlebermodellen aus dem Alten Orient. Neukirchen / Vluyn: Neukirchener Verlag.

Neu, Erich. 1996. Das hurritische Epos der Freilassung I. Untersuchungen zu einem hurritisch-hethitischen Textensemble aus Hattuša (Studien zu den Boğazköy-Texten 32). Wiesbaden: Harrassowitz.

Richter, Thomas. 2012. Bibliographisches Glossar des Hurritischen. Wiesbaden: Harrassowitz.

Rieken, Elisabeth. 2011. Einführung in die hethitische Sprache und Schrift. Unter Mitwirkung von U. Gradmann und J. Lorenz (Lehrbücher orientalischer Sprachen I/2). Münster: Ugarit-Verlag.

Rieken, Elisabeth (ed.). 2019. Keilschrifttafeln aus Kayalıpınar 1. Textfunde aus den Jahren 1999-2017. Wiesbaden: Harrassowitz.

Rieken, Elisabeth, Ilya Yakubovich. Ms. Encounters between Scripts in Bronze Age Asia Minor. In: Ilona Zsolnay (ed.). Seen Not Heard: Composition, Iconicity, and the Classifier Systems of Logosyllabic Scripts (in print).

Riemschneider, Kaspar K. 2004. Die akkadischen und hethitischen Omentexte aus Boğazköy (Dresdner Beiträge zur Hethitologie 12). Dresden: Verlag der TU Dresden. 
Salvini, Mirjo. 1980. Ittita e hurrico nei rituali di Boğazköy. Vicino Oriente 3: 153-167.

Salvini, Mirjo. 1988. Un texte hourrite nommant Zimrilim. Revue d'Assyriologie 82: 59-69.

Salvini, Mirjo. 1996. The Habiru prism of king Tunip-Teššup of Tikunani. Roma: Istituti editoriali e poligrafici internazionali.

Salvini, Mirjo. 2008. Corpus dei testi urartei I, II, III. Le iscrizioni su pietra e roccia. Roma: CNR.

Salvini, Mirjo. 2012. Corpus dei testi urartei, IV. Iscrizioni su bronzi, argilla e altri supporti. Nuove iscrizioni su pietra. Paleografia generale. Roma: CNR.

Salvini, Mirjo. 2015. Les textes hourrites de Meskéné/Emar. Analecta Orientalia 57 (2 vols.). Roma: Gregorian \& Biblical Press.

Salvini, Mirjo, Ilse Wegner. 1986. Die Rituale des AZU-Priesters (Corpus der hurritischen Sprachdenkmäler I, Band 2). Roma: Multigrafica editrice.

Salvini, Mirjo, Ilse Wegner. 2004. Die mythologischen Texte (Corpus der hurritischen Sprachdenkmäler I, Band 6). Roma: CNR.

Schwemer, Daniel. 1995. Das alttestamentliche Doppelritual 'lwt wšlmnym im Horizont der hurritischen Opfertermini ambašši und keldi. In: D. I. Owen, G. Wilhelm (eds.). Edith Porada Memorial Volume. Studies on the Civilization and Culture of Nuzi and the Hurrians 7: 81-116. Bethesda.

Singer, Itamar. 2002. Hittite Prayers (Writings From The Ancient World 11). Atlanta: Society of Biblical Literature.

Soysal, Oğuz. 2004. Hattischer Wortschatz in hethitischer Textüberlieferung (Handbook of Oriental Studies I/74). Leiden: Brill.

Starke, Frank. 1979. Halmašuit im Anitta-Text und die hethitische Ideologie vom Königtum. Zeitschrift für Assyriologie 69: 47-120.

Thureau-Dangin, François. 1939. Tablettes hurrites provenant de Mâri. Revue d'Assyriologie 36: 1-28.

Trémouille, Marie-Claude. 2005. Texte verschiedenen Inhalts (Corpus der hurritischen Sprachdenkmäler I, Band 8). Roma: CNR.

Van den Hout, Theo. 2002. Another View of Hittite Literature. Eothen 11: 857-878.

Von Dassow, Eva. 2013. Piecing together the Song of Release. Journal of Cuneiform Studies 65: 127-162.

Waal, Willemjin. 2015. Hittite Diplomatics: Studies in Ancient Document Format and Record Management (Studien zu den Boğazköy-Texten 57). Wiesbaden: Harrassowitz.

Wegner, Ilse. 2007. Einführung in die hurritische Sprache. 2., überarb. Aufl. Wiesbaden: Harrassowitz.

Wegner, Ilse, Mirjo Salvini. 1991. Die hethitisch-hurritischen Ritualtafeln des (h)išuwa-Festes (Corpus der hurritischen Sprachdenkmäler I, Band 4). Roma: Multigrafica editrice.

Wilhelm, Gernot. 1997. Kuşaklı - Sarissa Bd. 1/1: Keilschrifttexte aus Gebäude A. Rahden/Westf.: Marie Leidorf.

Wilhelm, Gernot. 2019. Die hurritischen Texte aus Šamuha. In: Elisabeth Rieken (ed.). Keilschrifttafeln aus Kayalıptnar 1. Textfunde aus den Jahren 1999-2017: 197-209. Wiesbaden: Harrassowitz.

Сузанна Гёрке. Гетерограммы в хурритских текстах из Богазкёя и Северной Сирии

В настоящей статье обсуждаются гетерографические написания в хурритских текстах второго тысячелетия до н.э. из Анатолии и северной Сирии. Частота шумеро- и аккадограмм анализируется в зависимости от жанра хурритских текстов. Большинство текстов религиозного содержания, включая ритуалы, описания праздников и мифы, содержат лишь небольшое количество логограмм и детерминативов. Исключением являются хурритские гадательные тексты из Хаттусы и Эмара, в которых гетерограммы используются достаточно часто. Для интерпретации полученных результатов необходимо использовать различные параметры, такие как степень усвоения иностранного языка писцами, возможные адресаты текстов, а также прагматика различных жанров и стилей. Для сравнения приводится краткий обзор гетерографических написаний в урартских текстах.

Ключевые слова: хурритские клинописные тексты; Анатолия; северная Сирия; урартские надписи; писцовые традиции. 